University of New Hampshire

University of New Hampshire Scholars' Repository

Physics Scholarship

Physics

7-1-2006

\title{
Magnetospheric influence on the Moon's exosphere
}

Jody K. Wilson

jody.wilson@unh.edu

M. Mendillo

Harlan E. Spence

Boston University, harlan.spence@unh.edu

Follow this and additional works at: https://scholars.unh.edu/physics_facpub

Part of the Physics Commons

\section{Recommended Citation}

Wilson, J. K., M. Mendillo, and H. E. Spence (2006), Magnetospheric influence on the Moon's exosphere, J. Geophys. Res., 111, A07207, doi:10.1029/2005JA011364.

This Article is brought to you for free and open access by the Physics at University of New Hampshire Scholars' Repository. It has been accepted for inclusion in Physics Scholarship by an authorized administrator of University of New Hampshire Scholars' Repository. For more information, please contact Scholarly.Communication@unh.edu. 


\title{
Magnetospheric influence on the Moon's exosphere
}

\author{
Jody K. Wilson, ${ }^{1}$ Michael Mendillo, ${ }^{1}$ and Harlan E. Spence ${ }^{1}$ \\ Received 15 August 2005; revised 31 January 2006; accepted 17 April 2006; published 26 July 2006.
}

[1] Atoms in the thin lunar exosphere are liberated from the Moon's regolith by some combination of sunlight, plasma, and meteorite impact. We have observed exospheric sodium, a useful tracer species, on five nights of full Moon in order to test the effect of shielding the lunar surface from the solar wind plasma by the Earth's magnetosphere. These observations, conducted under the dark sky conditions of lunar eclipses, have turned out to be tests of the differential effects of energetic particle populations that strike the Moon's surface when it is in the magnetotail. We find that the brightness of the lunar sodium exosphere at full Moon is correlated with the Moon's passage through the Earth's magnetotail plasma sheet. This suggests that omnipresent exospheric sources (sunlight or micrometeors) are augmented by variable plasma impact sources in the solar wind and Earth's magnetotail.

Citation: Wilson, J. K., M. Mendillo, and H. E. Spence (2006), Magnetospheric influence on the Moon's exosphere, J. Geophys. Res., 111, A07207, doi:10.1029/2005JA011364.

\section{Introduction}

[2] The impact of charged particles upon a surface has been a fundamental process of study in space plasma science. Referred to as space-weathering, the effect has been of direct relevance to aerospace engineering concerns about spacecraft component degradation, and of fundamental scientific interest in the liberation of surface material from the regoliths of celestial bodies. Theoretical work and laboratory experiments have shown that both ions (sputtering) and electrons (impact-stimulated desorption) have roles as space weathering agents [e.g., Johnson, 1990; Madey et al., 1998; Yakshinskiy and Madey, 1999, 2004]. In this study we use the Moon as a nearby laboratory-in-space to explore the role of magnetospheric energetic particle-impact as a mechanism for the production of atmospheric gases.

[3] The possibility that the Moon might have an atmosphere was a topic of early scientific interest. Both Galileo and Kepler sought to describe it. The post-Apollo era of lunar atmospheric science started with the detection of weak emissions from sodium $(\mathrm{Na})$ and potassium $(\mathrm{K})$ by Potter and Morgan [1988] and Tyler et al. [1988]. A comprehensive review of the field was made by Stern [1999]. Briefly, three types of sources have been considered as potential agents for the production of lunar gases: sunlight, solar wind particles, and micrometeors. The ejection speeds of atoms and molecules liberated from a surface by these agents can be comparable to the Moon's escape speed $(\sim 2.4 \mathrm{~km} / \mathrm{s})$, and thus a substantial population can form an exosphere and escape from the Moon altogether. This has given rise to the term "surface-boundary-exosphere" (SBE) by Stern [1999] as a distinct class of upper atmospheres in

\footnotetext{
${ }^{-1}$ Center for Space Physics, Boston University, Boston, Massachusetts, USA.

Copyright 2006 by the American Geophysical Union. 0148-0227/06/2005JA011364
}

the solar system. Bodies with gravitational fields comparable to or lower than the Moon include Mercury, asteroids, comets, and the satellites of Mars and the giant planets; thus SBE research has applications beyond the lunar case treated here. The proximity of the Moon to the Earth and its monthly orbital period provide unique opportunities to explore the spatial and temporal variations of charged particle populations reaching the lunar surface, and hence offer insights into natural charged-particle impact mechanisms that are not so easily studied at other locations.

\section{Tests for Sources of the Lunar Exosphere}

[4] One of best ways to test the importance of an exospheric source agent is to observe the lunar exosphere during both high and low states of the agent in question. For instance, a test for ultraviolet photon-stimulated desorption (PSD) might involve observing the exosphere once during quiet solar conditions and once during a large solar flare. Unfortunately, solar flares are difficult to predict in advance, and they persist for only a short period when they do occur, so such a test would require many observations and some luck.

[5] Meteor showers are the most obvious opportunities for testing the micrometeor-impact source of the lunar exosphere, with the exosphere expected to be denser immediately following the onset of a shower. The unusually spectacular 1998 Leonid meteor shower provided the first measurable increase in the sodium exosphere from meteor impact vaporization [Smith et al., 1999; Wilson et al., 1999]; however, such strong showers are very rare and not always quantitatively predictable. Other attempts to measure exospheric increases from meteor showers were inconclusive [Hunten et al., 1991; Cremonese and Verani, 1997; Verani et al., 1998].

[6] Useful tests for solar-wind ion sputtering and electron-stimulated desorption (ESD) are easier to come by. As 


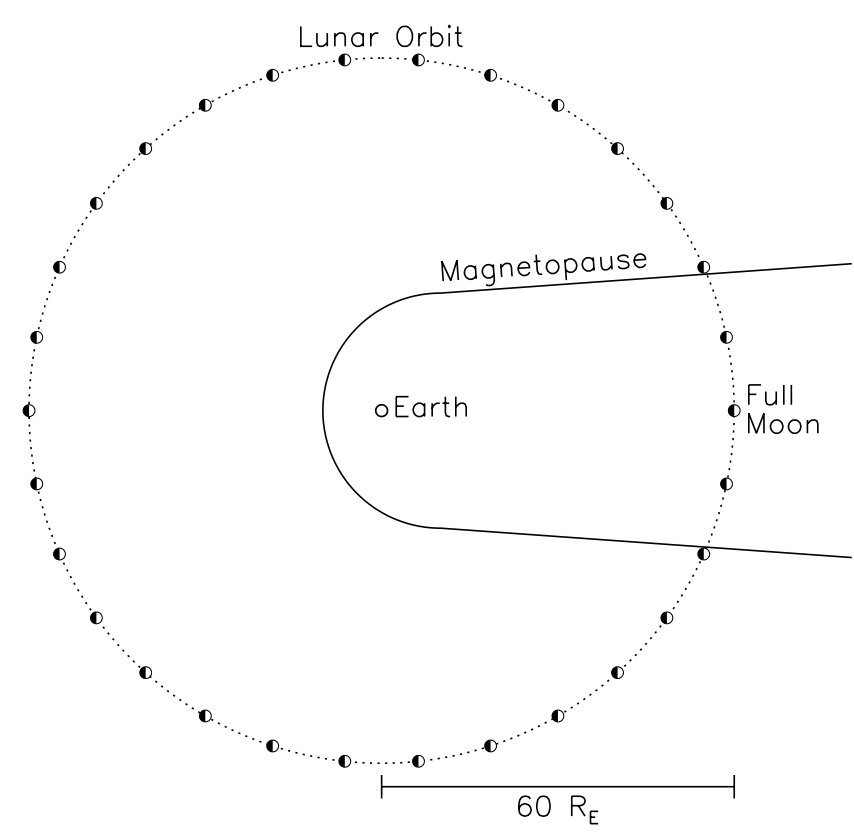

Figure 1. Geometry of the magnetotail and lunar orbit. The Moon's location in its orbit is shown at 1-day intervals. The Earth, lunar orbit, and approximate magnetotail boundary are drawn to scale. The Moon is not drawn to scale.

illustrated in Figure 1, the orbit of the Moon takes it through the Earth's magnetosphere once each month, traversing the geomagnetic tail at a distance of $\sim 60$ Earth radii $\left(\mathrm{R}_{\mathrm{E}}\right)$ for nearly 4 days. In the magnetotail the Moon is shielded from the solar wind, thus halting any sputtering or ESD caused by the solar wind. Potter and Morgan [1991] first suggested this as a possible test of the sputtering source; however, their initial results [Potter and Morgan, 1994] and a subsequent study [Potter et al., 2000] have left the issue unresolved.

[7] Observations of the lunar exosphere are uniquely difficult when the Moon is in the magnetotail because the Moon is near full phase and the sunlight reflected off of its surface is at its brightest. This results in high scattered light both in the Earth's atmosphere and in the sensitive instruments needed to observe the sodium emission. Our solution is to conduct imaging observations during the totality phase of a lunar eclipse, when for approximately 1 hour the Moon is within the umbra of the Earth's shadow and sunlight scattered off of the surface is vastly reduced. Since $\mathrm{Na}$ is detected through its resonant scattering of sunlight, the reduced photon flux in the Earth's umbra and penumbra limits detections close to the Moon. However, at distances beyond a few lunar radii $\left(\mathrm{R}_{\mathrm{M}}\right)$ observations are ultimately improved by the low scattered light, making the lunar exosphere detectable over a wide range of distances and brightness. Most of the $\mathrm{Na}$ atoms captured in this field of view are escaping from the Moon, and thus lunar eclipse observations offer unambiguous evidence of mechanisms responsible for the high-speed component of the Moon's surface-bounded exosphere [Wilson et al., 2003].

\section{Eclipse Observations of the Lunar Exosphere}

[8] The first wide-field imaging of the Moon's exosphere in eclipse [Mendillo and Baumgardner, 1995] showed a broad spatial region $\left(>6 \mathrm{R}_{\mathrm{M}}\right)$ of extended $\mathrm{Na}$ emission, indicating that the magnetospheric shielding of the Moon's surface from solar wind impact did not eliminate the exosphere. In other words, some process other than solar wind sputtering or solar wind ESD was contributing to the exosphere.

[9] It is important to note that, ironically, eclipse observations of the lunar exosphere are not sensitive to shadowing of photon-induced sources. The Moon has been in the umbra for typically $\sim 1$ hour at the time the images are taken, and $\mathrm{Na}$ escaping from the surface at $\sim 2 \mathrm{~km} / \mathrm{s}$ only travels $\sim 4 R_{M}$ in that time. Our measurements begin at $5 R_{M}$ from the Moon and extend to $12 \mathrm{R}_{\mathrm{M}}$ or more, beyond the range where the exosphere would be depleted due to a temporary decrease in PSD.

[10] Following the Mendillo and Baumgardner [1995] observation, three subsequent eclipses provided confirmation [Mendillo et al., 1999] and a fifth event documented the full extent of the lunar exosphere to distances of $\sim 20 \mathrm{R}_{\mathrm{M}}$ [Wilson et al., 2003]. In Table 1 we list the dates of the eclipses with the observational sites and solar and geophysical conditions. In Figure 2, a composite image from all five events is shown, together with radial profiles of the brightness versus distance for each event. Note that the first four observations were made with a $7^{\circ}$ field-of-view (FOV) camera system, which does not image the outer limit of the lunar exosphere. In the fifth event, a $60^{\circ} \mathrm{FOV}$ system was used, showing that the brightness asymptotically approaches zero beyond $\sim 20 \mathrm{R}_{\mathrm{M}}$.

[11] A second characteristic shown in Figure 2 is that the exospheric brightness is never the same. The five eclipse exospheres can be crudely grouped into two categories: dim

Table 1. Lunar Eclipse Conditions

\begin{tabular}{|c|c|c|c|c|c|c|c|c|c|c|}
\hline Date & UT & Site $^{\mathrm{a}} / \mathrm{CCD}^{\mathrm{b}}$ & Altitude $^{\mathrm{c}}$ & $\mathrm{Kp}$ & DST & $\mathrm{V}_{\mathrm{SW}}, \mathrm{km} / \mathrm{s}$ & Abberration $^{\mathrm{d}}$ & $\mathrm{B}_{\mathrm{Y}}, \mathrm{nT}$ & F10.7 & Previous Solar Flares \\
\hline 29 Nov 1993 & 0626 & $\mathrm{M} / \mathrm{s}$ & $80^{\circ}$ & 2 & -10 & 450 & $3.8^{\circ}$ & -3 & 94 & $\mathrm{C} 1,3 \mathrm{~h}$ \\
\hline 4 Apr 1996 & 0010 & $\mathrm{~L} / \mathrm{i}$ & $55^{\circ}$ & 3 & -20 & 370 & $4.7^{\circ}$ & -1 & 70 & None \\
\hline 27 Sep 1996 & 0254 & $\mathrm{M} / \mathrm{s}$ & $27^{\circ}$ & 4 & -40 & 650 & $2.6^{\circ}$ & -1 & 70 & None \\
\hline 24 Mar 1997 & 0439 & $\mathrm{M} / \mathrm{i}$ & $43^{\circ}$ & 1 & -10 & 350 & $4.9^{\circ}$ & -2 & 73 & None \\
\hline 16 Jul 2000 & 1355 & $\mathrm{~A} / \mathrm{i}$ & $80^{\circ}$ & 5 & -150 & 800 & $2.1^{\circ}$ & 0 & 225 & $\mathrm{M} 4,12 \mathrm{~h} \mathrm{X} 6,52 \mathrm{~h}$ \\
\hline
\end{tabular}

${ }^{a}$ M: McDonald Observatory, Texas; L: Las Palma, Canary Islands; A: Cairns, Australia.

${ }^{\mathrm{b}}$ Here s: standard CCD; i: intensified CCD.

${ }^{\mathrm{c}}$ Altitude of Moon above horizon at observing site.

${ }^{\mathrm{d}}$ Westward aberration angle of magnetotail. 

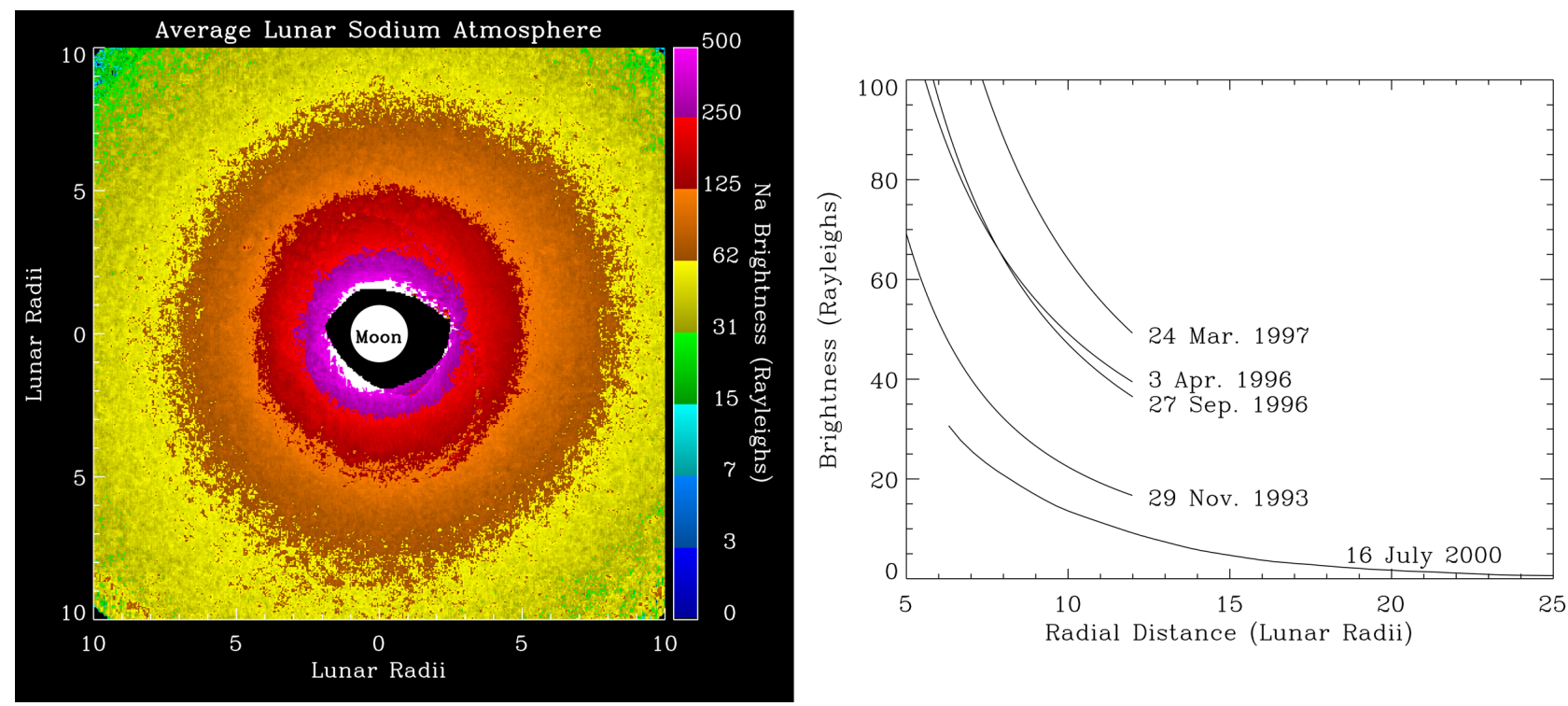

Figure 2. (left) Image of average lunar sodium exosphere during eclipse. (right) Individual radial profiles for each of the five lunar eclipse sodium exospheres.

(November 1993, July 2000) and bright (the remaining three).

\section{Solar Activity and Photodesorption}

[12] As shown in Table 1, the eclipses of November 1993 and July 2000 had the highest F10.7 values and were the only ones which occurred following solar flares. Higher UV fluxes, whether occurring near solar maximum or temporarily in a solar flare, are expected to increase the photodesorption rate of sodium atoms from the lunar surface. However, as shown in Figure 2, these two eclipses had the two dimmest exospheres observed. It is unlikely that enhanced photoionization of the exosphere accounts for the difference, since the photoionization lifetime of $\mathrm{Na}$ varies by less than $10 \%$ between active Sun and quiet Sun conditions [Huebner, 1992]. Unless UV photons are actually capable of reducing the release rate of sodium atoms from the surface for many hours, we must conclude that the higher UV fluxes in November 1993 and July 2000 did not significantly affect the exospheres observed during eclipse.

\section{Magnetospheric Populations and Geometry}

[13] Since there were no major meteor showers during any of the observations and since the Moon was shielded from the solar wind, it follows that changes in the magnetotail must somehow account for the difference between eclipse exospheres. The most obvious culprit is the relatively narrow and dense plasma sheet that lies between the two rarified lobes of the magnetotail. The plasma sheet in the vicinity of the Moon's orbit is approximately 5 Earth radii thick, and it is less dense but more energetic than the solar wind plasma [Rich et al., 1973]. The lobe plasma is less dense and cooler than the plasma sheet [Frank, 1985]. Thus near full Moon, the Moon usually resides in the cooler and more rarified plasma of the lobes but briefly encounters hotter and denser plasma during plasma-sheet crossings. These crossings may temporarily augment the exosphere via ion sputtering, ESD, or other plasma impact effects. Table 2 summarizes the different plasma populations encountered by the Moon.

[14] The location of the plasma sheet in the magnetotail depends largely on the orientations of the Earth's magnetic poles relative to the Sun but also on solar wind properties such as speed, density, and magnetic field strength and direction. Tsyganenko et al. [1998] have produced an empirical model of the Earth's magnetotail from spacecraft data that predicts the plasma sheet location at the Moon's distance of $60 \mathrm{R}_{\mathrm{E}}$. (More recent models also exist, but none extends to the Moon's distance.) We use this model to predict the location of the plasma sheet during each of the five eclipse periods, thereby estimating plasma sheet-crossing times for the Moon before each eclipse.

[15] The eclipse of 16 July 2000 occurred during a large geomagnetic storm, meaning the Tsyganenko model may not be an accurate predictor for that case. Rich et al. [1973] found that higher geomagnetic activity reduced the probability of the Moon encountering the plasma sheet due either to a thinning of the plasma sheet or to a large displacement of the plasma sheet from its location during quiet conditions. We use the Tsyganenko model for the July 2000

Table 2. Plasma Conditions in Three Regimes

\begin{tabular}{|c|c|c|c|}
\hline & $\begin{array}{c}\text { Ion Energy, } \\
\mathrm{eV}\end{array}$ & $\begin{array}{c}\text { Electron Energy, } \\
\mathrm{eV}\end{array}$ & $\begin{array}{c}\text { Density, } \\
\mathrm{cm}^{-3}\end{array}$ \\
\hline Solar Wind & $200-3000{\text { (flow })^{\mathrm{a}}}^{\mathrm{a}}$ & $5-25^{b}$ & $1-10^{b}$ \\
\hline Plasma Sheet ${ }^{\mathrm{c}}$ & $1000-5000$ & $175-325$ & $\sim 0.1$ \\
\hline Magnetotail Lobes $^{\mathrm{b}}$ & $<1000$ & $<100$ & $\sim 0.01$ \\
\hline
\end{tabular}

${ }^{\mathrm{a}}$ Flow speeds are higher than thermal ion speeds.

${ }^{\mathrm{b}}$ Frank [1985].

${ }^{\mathrm{c}}$ Rich et al. [1973]. 

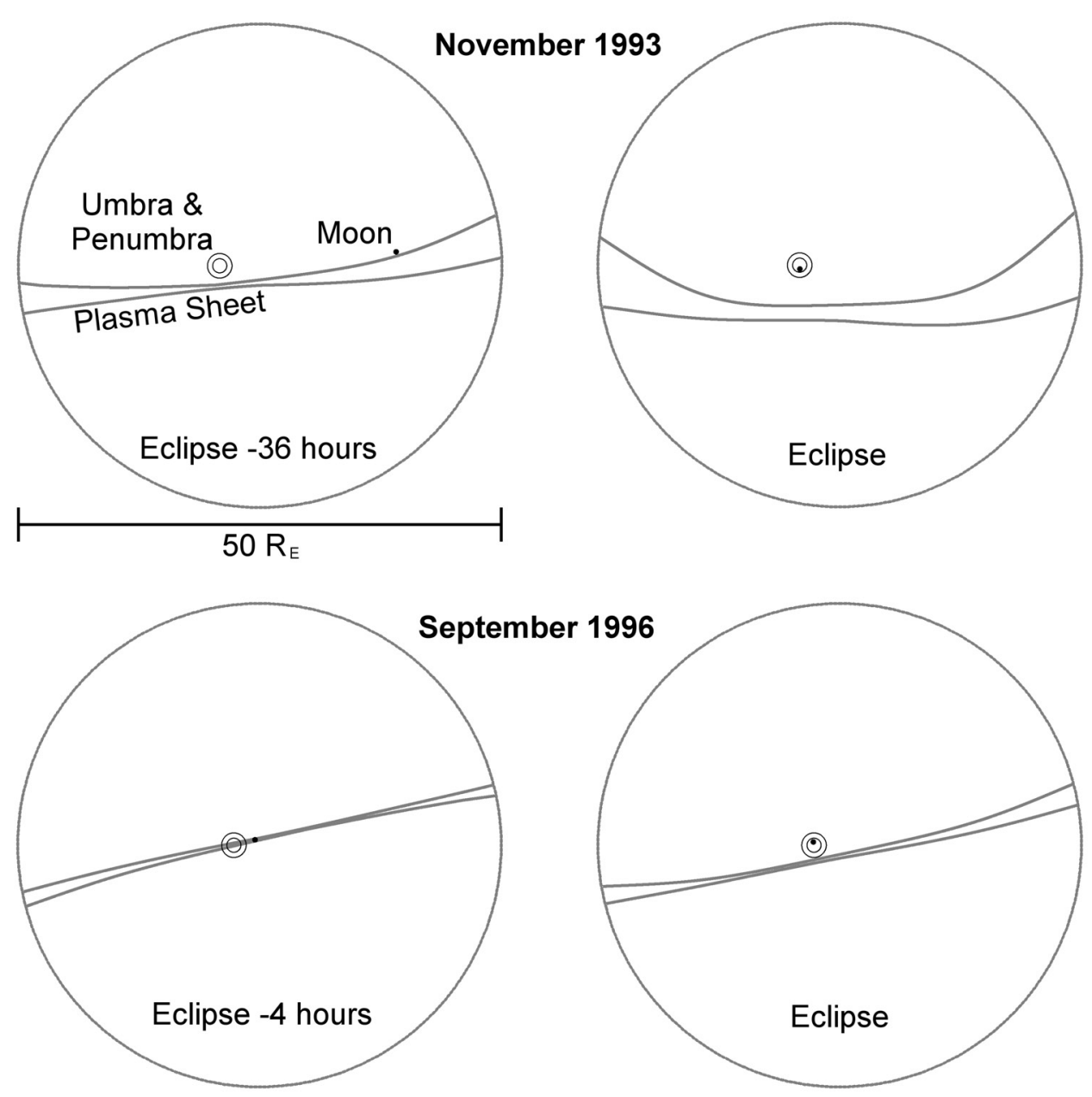

Figure 3. Examples of locating the Moon within the cross section of the Tsyganenko magnetotail model for (top) November 1993 and (bottom) September 1996. The most northerly and most southerly possible plasma sheet locations are indicated by the two solid lines in each plot, based on the uncertainties in the Tsyganenko model. In November 1993, the Moon's last possible plasma sheet crossing (top left) occurred 36 hours before it was imaged in eclipse (top right). However, in September 1996, the Moon's last plasma sheet crossing (bottom left) occurred only 4 hours before being imaged in eclipse (bottom right).

eclipse anyways, while being mindful of the findings of Rich et al.

[16] The Tsyganenko et al. [1998] model uses the $\mathrm{B}_{\mathrm{Y}}$ component of the solar wind and the Earth's magnetic field orientation relative to the Sun to predict the two-dimensional orientation of the plasma sheet within cross-tail planes at several distances down the magnetotail. Since the Moon's distance of $60 \mathrm{R}_{\mathrm{E}}$ is actually the dividing line between two output bins in the model (40-60 $R_{E}$ and $60-100 R_{E}$ ), we use the average outputs from those two output bins. We include the slight westward aberration of the magnetotail that results from the solar wind speed (from 350 to $800 \mathrm{~km} / \mathrm{s}$ ) and the Earth's orbital speed $(30 \mathrm{~km} / \mathrm{s})$ perpendicular to the solar wind; this shifts the magnetotail $2^{\circ}-5^{\circ}$ from the EarthSun line or $2-5 \mathrm{R}_{\mathrm{E}}$ west at the Moon's distance. Figure 3 shows examples of output from the Tsyganenko et al. model superposed with the locations of the Moon and the Earth's shadow. Figure 4 shows plots of the distance of the Moon from the plasma sheet for all five preeclipse periods.

\section{Analysis and Discussion}

[17] There is a significant difference in the Moon-plasmasheet distance for the five preeclipse periods. The two dim eclipse exospheres of November 1993 and July 2000 occurred more than 30 hours after the most recent plasma sheet crossings. The three bright eclipse exospheres, by contrast, occurred less than 15 hours after the most recent crossing. This is shown more clearly in Figure 5, where the exosphere brightness is plotted versus plasma sheet crossing time.

[18] Interestingly, the eclipse exosphere of July 2000, which was imaged during a strong geomagnetic storm, was the dimmest of the five exospheres observed. The findings of Rich et al. [1973] indicate that the Moon was 
Moon - Plasma Sheet Distance
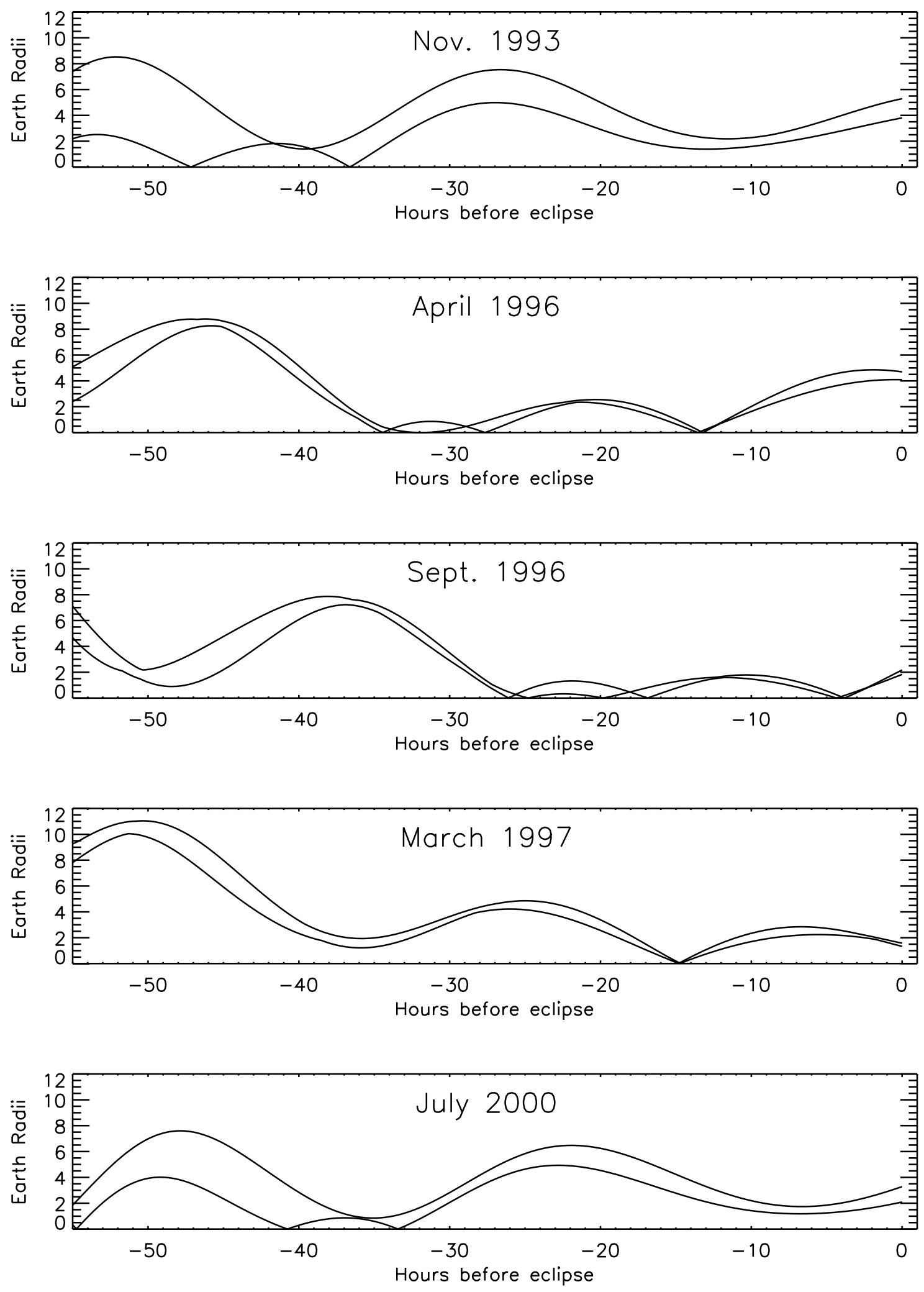

Figure 4. Plots of Moon-to-plasma-sheet distance versus time for all five preeclipse periods. To demonstrate the uncertainties in the Tsyganenko model, two lines are plotted for each period, representing the most northern possible and most southern possible plasma sheet locations. In November 1993 and July 2000, the most recent plasma sheet crossings occurred more than 30 hours before mideclipse (if they occurred at all), while plasma sheet crossings occurred less than 15 hours from mideclipse for the other three events. 


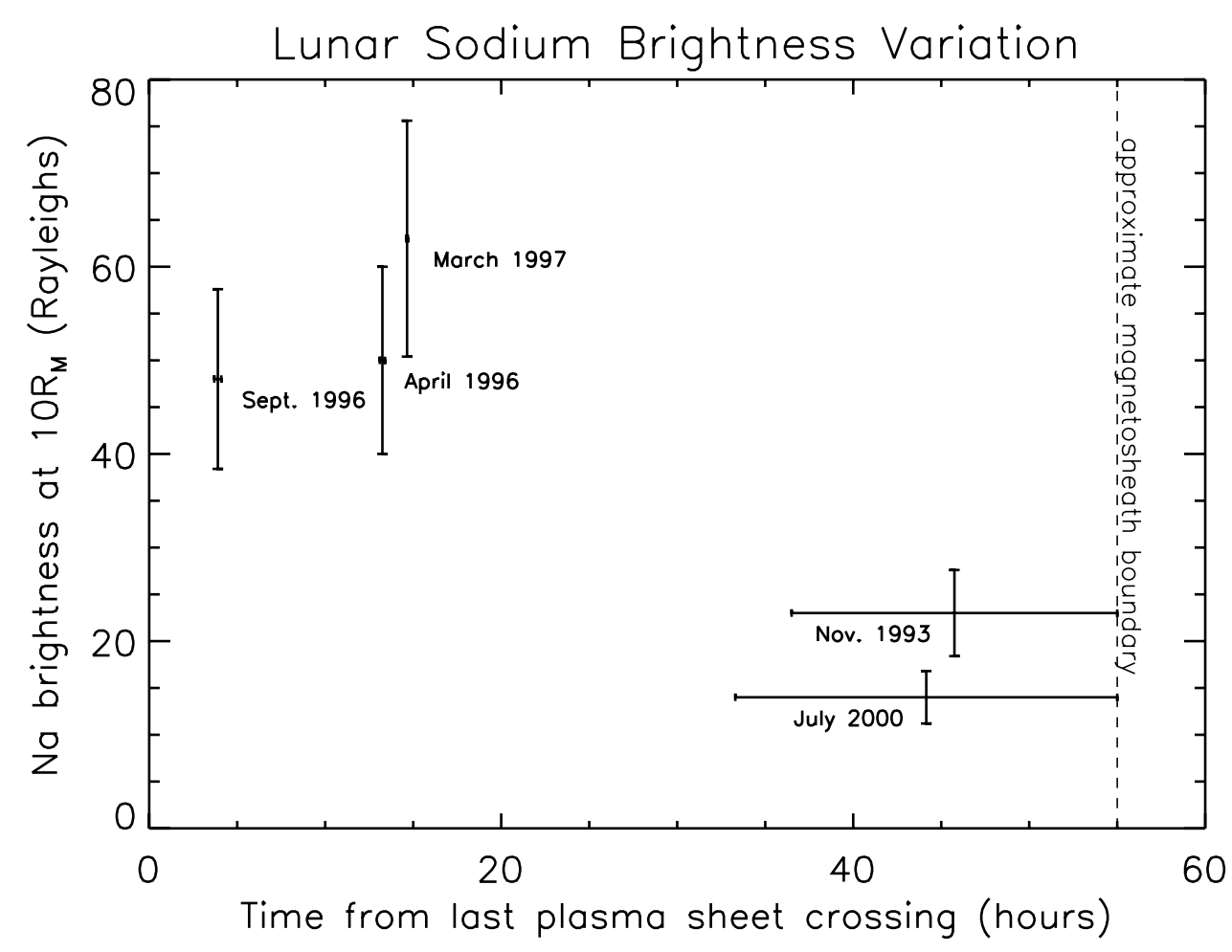

Figure 5. Lunar sodium exosphere brightness versus time of most recent plasma sheet crossing. The error bars in crossing time represent the uncertainties in the Tsyganenko model, and the error bars in $\mathrm{Na}$ brightness are due to standard star calibration uncertainties. (Note that the crossing time error bars for the brightest three eclipses are very small.) For the dimmest two eclipses, the Tsyganenko model predicts that there may have been no plasma sheet crossing at all prior to eclipse; in those cases the most recent exposure to hot and dense plasma would have been in the magnetosheath, which the Moon passes out of approximately 55 hours before eclipse, as reflected in the crossing time error bars.

even less likely to have encountered the plasma sheet than the Tsyganenko model predicts for that period. That would suggest that the Moon last encountered dense, hot plasma in the magnetosheath, a case which would fit very well with the observed trend.

[19] The trend shown in Figure 5 suggests that plasma sheet constituents impacting the lunar surface increase the rate of sodium outgassing from the lunar surface into the exosphere. What is surprising is that up to 15 hours after the plasma sheet passage, the exosphere is still significantly brighter than it is 30 hours later. This means that ion sputtering/electron desorption followed by immediate escape does not fully describe the effect of the plasma sheet. Somehow the impact of plasma sheet populations upon the lunar surface affects the flux of atoms leaving the surface for many hours after the plasma impact has ceased.

[20] At this point we would like to emphasize that the plasma sheet population is not necessarily a perfect replacement for the solar wind; the solar wind is typically denser by a factor of 10 or more as shown in Table 2 . However, plasma sheet particles are more energetic than their solar wind counterparts, particularly so for the electrons, so the sputtering and desorption yields per particle are probably higher in the plasma sheet than in the solar wind. Regardless, the more important distinction for this exospheric data set is between the plasma sheet (denser and hotter) and lobe plasma (cooler and sparser), where there can be little doubt that the former must sputter and desorb at higher rates than the latter.

[21] The delayed reaction of sodium escape following bombardment by charged particles in the plasma sheet could have any of three possible causes. First, newly sputtered/ desorbed $\mathrm{Na}$ atoms may not reach the escaping regions of the exosphere immediately. Low-energy (and low-altitude) exospheric $\mathrm{Na}$ may be readsorbed on the lunar surface where it is later released at higher energies by a different mechanism. This would be consistent with the suggestion of Kozlowski et al. [1991] and Sprague et al. [1992] that newly liberated sodium atoms quickly readsorb to the surface away from the subsolar point where they are gradually released (at higher speeds) by photodesorption. In the case of eclipse observations, this delayed photodesorption contribution would begin just after the plasma sheet passage and temporarily stop in the Earth's umbra just before our images are taken.

[22] Second, the effect of impacting plasma may be to "garden" the lunar surface microscopically, driving diffusion of $\mathrm{Na}$ locked within the soil toward the surface where it can ultimately escape into the exosphere. McGrath et al. [1986] suggested such a "radiation-enhanced diffusion" source for Mercury's $\mathrm{Na}$ exosphere. The lunar data here would suggest that the effects of radiation-enhanced diffusion last many hours after the radiation ceases. This might be explained if other agents (photons or micrometeors) 
make use of the temporarily higher $\mathrm{Na}$ densities near the surface.

[23] Third, the unique plasma properties in the plasma sheet may temporarily change the electrical charge state of the lunar surface [e.g., Reasoner et al., 1973], which is also capable of altering the $\mathrm{Na}$ diffusion rate in the lunar soil [Madey et al., 1998]. As with radiation-enhanced diffusion, this may temporarily increase the $\mathrm{Na}$ abundance in the top of the lunar soil in some regions, allowing for temporarily higher $\mathrm{Na}$ yields from other source processes.

\section{Conclusions}

[24] The observed effect of plasma sheet passage on the lunar sodium exosphere implies that plasma impact on the Moon does indeed contribute to the production of the lunar exosphere. However, the exact mechanism at work remains unclear. The time delay between plasma sheet passage and exospheric enhancement suggests at least one of three possible phenomena at work: (1) a delay between the initial liberation of $\mathrm{Na}$ atoms by sputtering or ESD and their appearance in the escaping regions of the exosphere, (2) radiation-enhanced diffusion of $\mathrm{Na}$ during plasma sheet passage, or (3) diffusion enhancement by surface charging.

[25] Acknowledgments. This research was supported by the Center for Space Physics and grants from the NASA Planetary Astronomy program. We would like to thank Bob Johnson and Nikolai Tsyganenko for helpful discussions and comments.

[26] Arthur Richmond thanks Robert E. Johnson and Theodore E. Madey for their assistance in evaluating this paper.

\section{References}

Cremonese, G., and S. Verani (1997), High resolution observations of the sodium emission from the Moon, Adv. Space Res., 19, 1561-1569.

Frank, L. A. (1985), Plasmas in the Earth's magnetotail, Space Sci. Rev., 42, 211-240.

Huebner, W. F. (1992), Solar photo rates for planetary atmospheres and atmospheric pollutants, Astrophys. Space Sci., 195, 1-294.

Hunten, D. M., R. W. H. Kozlowski, and A. L. Sprague (1991), A possible meteor shower on the Moon, Geophys. Res. Lett., 18, 2101-2104.

Johnson, R. E. (1990), Energetic Charged-Particle Interactions with Atmospheres and Surfaces, pp. 97-121, Springer, New York.

Kozlowski, R. W. H., A. L. Sprague, D. M. Hunten, W. K. Wells, and F. A. Grosse (1991), Sodium in the lunar atmosphere: Dependence of release mechanisms on local solar zenith angle, Bull. Am. Astron. Soc., 23, 1198.

Madey, T. E., B. V. Yakshinskiy, V. N. Ageev, and R. E. Johnson (1998), Desorption of alkali atoms and ions from oxide surfaces: Relevance to origins of $\mathrm{Na}$ and $\mathrm{K}$ in atmospheres of Mercury and the Moon, J. Geophys. Res., 103, 5873-5887.
McGrath, M. A., R. E. Johnson, and L. J. Lanzerotti (1986), Sputtering of sodium on the planet Mercury, Nature, 323, 694-696.

Mendillo, M., and J. Baumgardner (1995), Constraints on the origin of the Moon's atmosphere from observations during a lunar eclipse, Nature, 377, 404.

Mendillo, M., J. Baumgardner, and J. K. Wilson (1999), Observational test for the solar wind origin of the Moon's sodium atmosphere, Icarus, 137, $13-23$.

Potter, A. E., and T. H. Morgan (1988), Discovery of sodium and potassium vapor in the atmosphere of the Moon, Science, 241, 675-680.

Potter, A. E., and T. H. Morgan (1991), Observations of the lunar sodium exosphere, Geophys. Res. Lett., 18, 2089-2092.

Potter, A. E., and T. H. Morgan (1994), Variations of lunar sodium emission intensity with phase angle, Geophys. Res. Lett., 21, 2263-2266.

Potter, A. E., R. M. Killen, and T. H. Morgan (2000), Variation of lunar sodium during passage of the Moon through Earth's magnetotail, J. Geophys. Res., 105, 15,073-15,084.

Reasoner, D. L., F. J. Rich, and W. J. Burke (1973), Interaction between the lunar photoelectron layer and the plasma sheet, Abstr. Lunar Planet. Sci. Conf., 4, 618 .

Rich, F. J., D. L. Reasoner, and W. J. Burke (1973), Plasma sheet at lunar distance: Characteristics and interactions with the lunar surface, J. Geophys. Res., 78, 8097-8112.

Smith, S. M., J. K. Wilson, J. Baumgardner, and M. Mendillo (1999), Discovery of the distant lunar sodium tail and its enhancement following the Leonid meteor shower of 1998, Geophys. Res. Lett., 26, 1649-1652.

Sprague, A. L., R. W. H. Kozlowski, D. M. Hunten, W. K. Wells, and F. A. Grosse (1992), The sodium and potassium atmosphere of the moon and its interaction with the surface, Icarus, 96, 27-42.

Stern, S. A. (1999), The lunar atmosphere: History, status, current problems, and context, Rev. Geophys., 37, 453-491.

Tsyganenko, N. A., S. B. P. Karlsson, S. Kokubun, T. Yamamoto, A. J. Lazarus, K. W. Ogilvie, C. T. Russell, and J. A. Slavin (1998), Global configuration of the magnetotail current sheet as derived from Geotail, Wind, IMP 8 and ISEE 1/2 data, J. Geophys. Res., 103, 6827-6842.

Tyler, A. L., R. W. H. Kozlowski, and D. M. Hunten (1988), Observations of sodium in the tenuous lunar atmosphere, Geophys. Res. Lett., 15 , $1141-1144$.

Verani, S., C. Barbieri, C. Benn, and G. Cremonese (1998), Possible detection of meteor stream effects on the lunar sodium atmosphere, Planet. Space Sci., 46, 1003-1006.

Wilson, J. K., S. M. Smith, J. Baumgardner, and M. Mendillo (1999), Modeling an enhancement of the lunar sodium atmosphere and tail during the Leonid meteor shower of 1998, Geophys. Res. Lett., 26, 1645-1648.

Wilson, J. K., J. Baumgardner, and M. Mendillo (2003), The outer limits of the lunar sodium exosphere, Geophys. Res. Lett., 30(12), 1635 , doi:10.1029/2003GL017085.

Yakshinskiy, B. V., and T. E. Madey (1999), Photon-stimulated desorption as a substantial source of sodium in the lunar atmosphere, Nature, 400, $642-644$.

Yakshinskiy, B. V., and T. E. Madey (2004), Photon-stimulated desorption of $\mathrm{Na}$ from a lunar sample: Temperature-dependent effects, Icarus, 168, $53-59$.

M. Mendillo, H. E. Spence, and J. K. Wilson, Center for Space Physics, Boston University, 725 Commonwealth Avenue, Boston, MA 02215, USA (jkwilson@bu.edu) 